\title{
Development of a Flexible and Disposable Microneedle-Fluidic-System With Finger-Driven Drug Loading and Delivery Functions for Inflammation Treatment
}

\author{
Zhuolin Xiang, Student Member, IEEE, Hao Wang, Giorgia Pastorin, and Chengkuo Lee, Member, IEEE
}

\begin{abstract}
Patch-based transdermal drug delivery technology offers a convenient way to administer drugs without the drawbacks of standard hypodermic injections related to issues, such as patient acceptability and injection safety. In this paper, we developed a flexible and disposable microneedle-fluidicsystem (MFS), which can achieve finger driven on-chip drug loading and delivery functions by integrating polydimethylsiloxane-based microfluidic dispensing networks, check valves, micropump, and SU-8 microneedles in a patch device. The control of particular loading or delivery volume for drugs is provided in terms of finger pressing to a micropump with a given dimension. The in vitro mechanical testing, penetration testing, and delivery testing prove the device functionalities. The local inflammation phenomenon of rats has been ameliorated with the aid of successful diclofenac deliver by our MFS patch.

[2015-0046]
\end{abstract}

Index Terms-Finger driven, local inflammation treatment, microneedles, personal healthcare device.

\section{INTRODUCTION}

D RUG DELIVERY research has been studied extensively for administering a pharmaceutical compound to achieve a therapeutic effect in humans or animals. The conventional drug delivery approaches, such as oral administration and hypodermic injection, have their own limitations because a drug may be inactive through phase I metabolism during oral delivery [1], while the hypodermic injection requires trained personnel [2]. In order to overcome these drawbacks, transdermal drug delivery has been taken as a safe, easily accessible and patient friendly approach [3], [4]. The normal biomolecules, however, have difficulties to passively diffuse through the dermal layers of skin due to their unfavorable hydrophilicity and macro size [5], [6]. With the help of

Manuscript received February 11, 2015; revised April 18, 2015; accepted May 2, 2015. Date of publication May 20, 2015; date of current version June 1, 2015. This work was supported by the Singapore National Research Foundation through the Project entitled Self-Powered Body Sensor Network for Disease Management and Prevention Oriented Healthcare under Grant R-263-000-A27-281. Subject Editor X. Zhang.

Z. Xiang, H. Wang, and C. Lee are with the Department of Electrical and Computer Engineering, National University of Singapore, Singapore 117576 (e-mail: a0086268@u.nus.edu; elewangh@nus.edu.sg; elelc@nus.edu.sg).

G. Pastorin is with the Department of Pharmacy, National University of Singapore, Singapore 117543 (e-mail: phapg@nus.edu.sg).

Color versions of one or more of the figures in this paper are available online at http://ieeexplore.ieee.org.

Digital Object Identifier 10.1109/JMEMS.2015.2429675 microneedles which can create microchannels on the skin surface by penetrating the outmost layer, i.e. stratum cornuem, not only the small molecules [7], [8] but also the high weight molecules can permeate into the tissue under the skin [9]-[12]. Since these microneedles are only a few hundred micrometers in length, the penetration depth is superficial enough not to touch nerve receptors in the lower reticular dermis, which leads the whole administration to be painless [13], [14]. It is promising that this microneedle-based transdermal delivery approach will offer a self-management, patient-friendly and efficient administration route for drug delivery.

Although the study of microneedles has been lasted for decades, most works focused on the novel fabrication technology of microneedles for penetration purpose [15]-[19] and the validation of drug formulations delivered by microneedle approaches [20]-[23]. However, microneedles still have some critical limitations which keep them from being widely used in the practical experiments and our daily life. Firstly, it would be particularly desirable for the microneedles chip to remain in the precise location and maintain the fluid communication with the tissues beneath the statum corneum for an extended period. Excepted for the consideration of micorneedle density [24] and optimized insertion force [25], flexibility of the microneedle patch is also a key feature to meet this requirement since the skin is contoured and deformable. Thus, the conventional microneedle patches with a rigid and planar substrate which are made by silicon [26], [27], stainless steel [28], [29] or nickel [30], [31] may encounter a difficulty to be attached tightly on the skin surface. Polymer microneedles patch has become attractive recently since one of its advantages is that the baseplate possess some degree of flexibility [32]-[34]. Although microneedles have been demonstrated to be transferred to a flexible substrate by molding technology [35], [36], the challenge of integrating these demolded microneedles into a miniaturized fluidic system limits their delivery volume. Moreover, in order to control transdermal delivery volume, extra syringes, tubes and syringe pumps usually need to be connected to the conventional microneedles chips for drug loading and delivery actuation purposes [37]-[39]. In this way, the usage of microneedles needs unfavorable additional components, which makes it not convenient to be used 


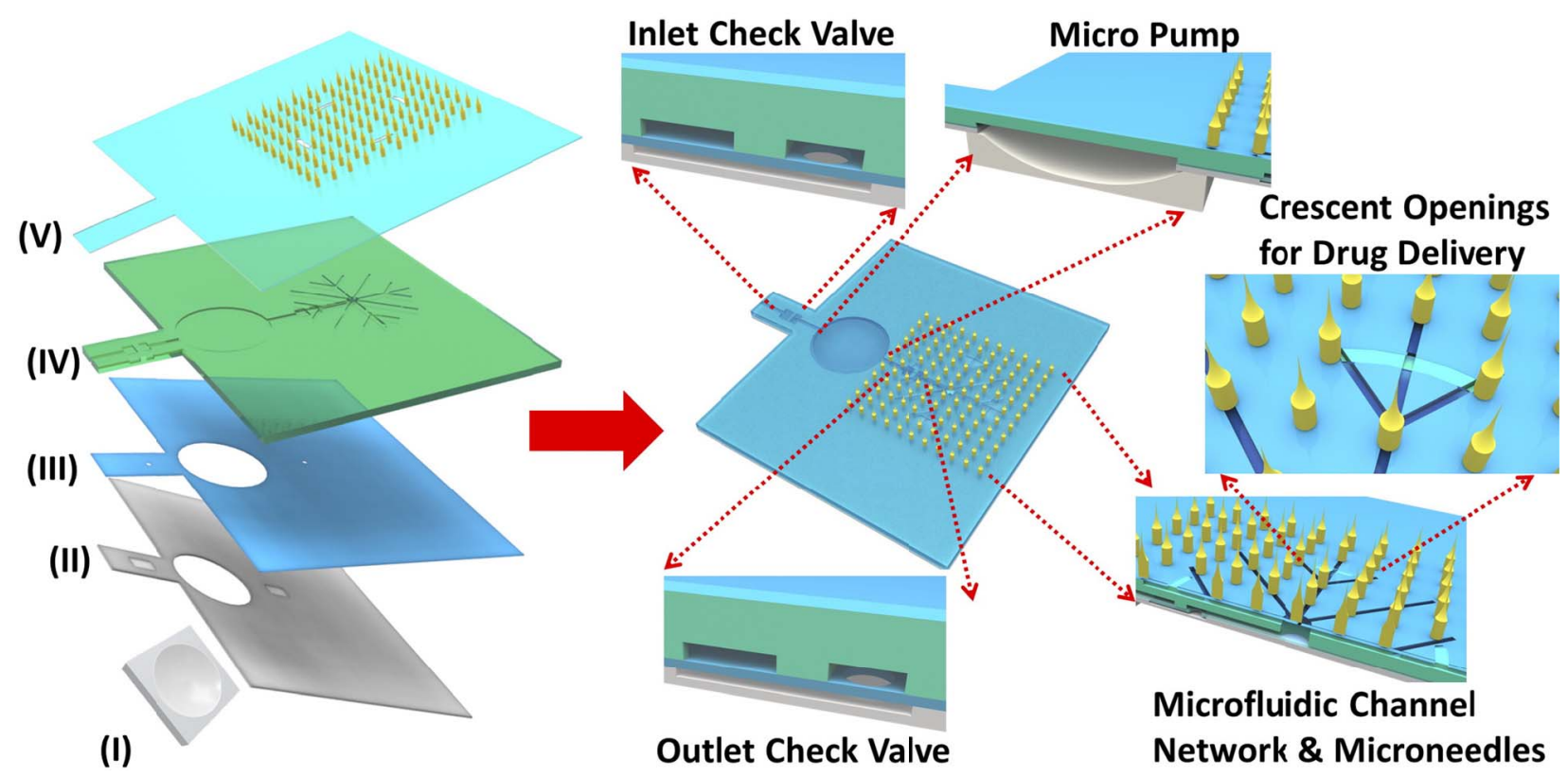

Fig. 1. A schematic depiction of Microneedle-Fluidic-System (MFS). Left-hand side is the explosive image for layer (I) to layer (V). The right-hand side images are detailed components in the MFS.

as personal healthcare devices. Even though several active control components, like PZT (piezoelectric) pump [40], electrical heater pump [41] and low-boiling-point liquid pump [42] have been integrated in the microneedles chips for drug dispensing control, these components combination required expensive materials and complicate fabrication process, which resulted in high costs and made them improper to be disposable devices. Low cost and passive micro pump has been widely used in microfluidic study, such as colorimetric tests for glucose and uric acid [43], encapsulation of endothelial cells in droplets [44], detection of hepatitis $C$ virus [45] and on-chip blood transportation [46]. However, there is no reported data of polymer based micro pump integrated in a microneedle device to control transdermal drug delivery volume. Furthermore, diclofenac is one of the most common non-steroidal anti-inflammatory drugs (NSAID) to treat various local inflammation phenomenon. The diclofenac is highly desired to be transdermal delivered due to its risk of cardiovascular events [47], [48] as well as serious potential life-threatening gastrointestinal bleeding [49], [50] when delivered through hypodermic injection or oral administration. Even through diclofeanc has been studied to delay micropore closure following microneedle treated skin such that naltrexone delivery is enhanced [51]-[53], there is no reported study which improves diclofenac transdermal delivery to ameliorate local inflammation phenomenon with the aid of microneedles.

In this study, we will demonstrate a flexible and disposable microneedle-fluidic-system (MFS) which can achieve finger driven on-chip drug loading and delivery functions by integrating Polydimethylsiloxane (PDMS) based microfluidic dispensing networks, check valves, micro pump and microneedles on a single device. The drug delivery can be actuated by finger pressing the integrated PDMS micro pump.
Compared with conventional microneedles devices, it is the first time to realize a flexible microneedle patch integrated with microfluidic components for on-chip loading and delivery control. The simplicity and low-cost nature of proposed MFS lead it to be a proper and promising disposable healthcare device. We also report the first in vivo experiment that the local inflammation phenomenon is treated and remedied with the aid of transdermal delivery of diclofenac solution into tissues using proposed MFS.

\section{Design And Fabrication}

The schematic drawing of the MFS is shown in Fig. 1 . The MFS is composed of five PDMS layers. Layer (I) is a hemisphere PDMS micro pump. It also acts as a drug storage chamber when the drug is loaded in the chip. A cavity and two through-holes are on the layer (II) and layer (III) respectively to form the check valves. The layer (IV) is a double-sided layer. The microfluidic channel at bottom side is bonded with the lower layer (III) to realize the check valve function while at top side there is another microfluidic channel network for drug dispensing purpose. The microfluidic channels at two sides are connected by a through-hole at the center of the microfluidic network. The layer $(\mathrm{V})$ is bonded on the top of layer (IV) as a cap for the microfluidic channel network. It has four crescent openings at the end of the microfluidic channel network for drug delivery purpose. A $16 \times 16$ SU-8 microneedles array is fabricated on the top of layer $(\mathrm{V})$. When the MFS is applied on the skin surface, these microneedles can penetrate the stratum cornuem layer and create microchannles in the tissue. Drugs loaded in the MFS by micro pump can be driven to pass the outlet check valve, flow to the top-side microfluidic network through the connection hole, dispense to microfluidic network branches and deliver through the crescent openings. These drugs then can permeate 


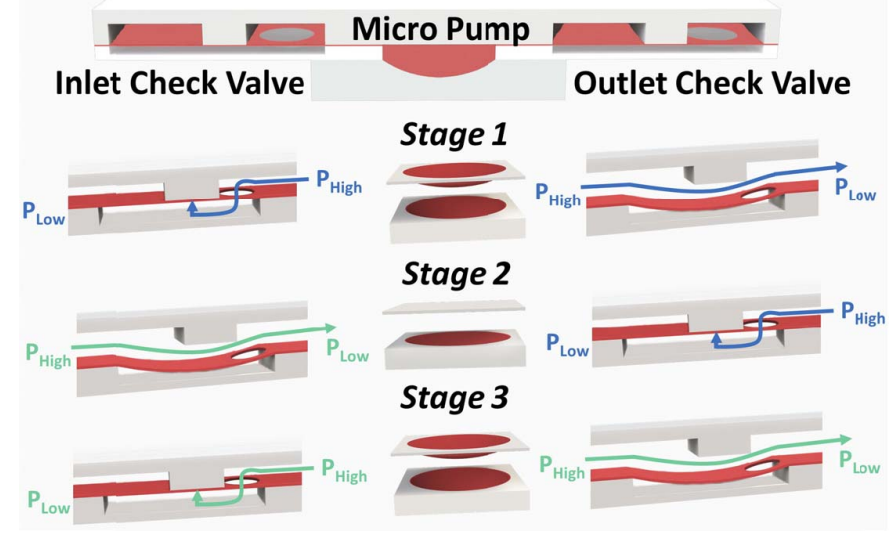

Fig. 2. Operation principle for the integrated check valves and micro pumps in MFS. The blue arrow represents air pressure while the green arrow represents fluid pressure.

through the microchannels created by microneedles and be delivered into tissues under the skin. Fig. 2 shows the operation principle for the integrated check valves and micro pump. The MFS has two check valves in series and the micro pump between them. Check valves only allow one directional flow acting as the flow diodes [54]-[57]. The two check valves, similar to the design reported by Mosadegh et al. [58], have three parts: an interrupted microchannel in one layer, a cavity in the other layer and a deformable membrane in between that can deflect into the cavity to allow the interrupted channel to become connected. Two serial check valves enable that the drug is loaded through one valve and delivered through the other. At the stage 1, after pressing the micro pump, the PDMS top layer deforms and gas inside is pushed out, which makes the pressure in the chamber become smaller than the air pressure outside. At stage 2, when the PDMS top layer is released, the drug is driven by the air pressure from the loading port and flows inside the chamber through inlet check valve. However, the outlet check valve is sealed by the gas pressure and the drug is kept inside the chamber. At stage 3, after pressing the micro pump again, drug inside the chamber can only be driven to flow through outlet check valve while the inlet check valve is sealed by the fluid pressure. In this way, the drug can be loaded inside the chip and delivered without any external components.

The detailed fabrication process for the MFS is shown in Fig. 3. All of PDMS structures in MFS are transferred from SU-8 masters. On the layer (I), a SU-8 droplet was deposited on a $50 \mu \mathrm{m}$ thick predefined SU-8 circular pedestal. After curing and UV lithography process, the SU-8 droplet master was cross linked (Fig. 3 (I a)). A hemisphere structure was molded using PDMS from this SU-8 droplet master (Fig. 3 (I c)). Then both layer (II) and layer (III) were fabricated by normal PDMS replica process from their respective SU-8 masters. Since layer (III) had two throughholes structures, a $100 \mu \mathrm{m}$ thick PDMS layer was deposited on its $350 \mu \mathrm{m}$ thick SU-8 pillars master. Meanwhile, a $300 \mu \mathrm{m}$ PDMS cavity structure on layer (II) was peeled off (Fig. 3 (II C)) and bonded with layer (III) (Fig. 3 (II + III a)).
After the bonded layer was released from the substrate (Fig. 3 (II + III b)), a hole with the same diameter as the circular pedestal on layer (I) was punched to be a part of final pressure chamber (Fig. 3 (II + III c)). Layer (IV) was a double-sided structure which contained both interrupted microfluidic channel at bottom side and microfluidic network branches at top side. Similar as a method reported in [59] and [60], this double-sided PDMS layer was fabricated by a molding process conducted in a clamping setup. The desired bottom side structure (Fig. 3 (IV a)) and top side structure (Fig. 3 (IV b)) were photo lithographically patterned as extruded SU-8 masters on two substrates respectively. Both masters were arranged in a double-sided molding clamping setup and the desired spacing between the masters was adjusted (Fig. 3 (IV c)). This spacing defined the thickness of the intermediate PDMS layer. For the vertical alignment of two patterned structures, both masters were provided with alignment structures in the peripheral regions. To ensure that all structure were completely covered with PDMS and that the PDMS was free of air bubbles, the clamping setup filled with PDMS was degassed in a vacuum chamber for 2 hours before PDMS curing. The double-sided PDMS layer was then peeled off from the SU-8 masters (Fig. 3 (IV d)) and bonded with layer (II) and layer (III) (Fig. 3 (II + III + IV)).

Four $350 \mu \mathrm{m}$ thick SU-8 crescent patterns were defined on a silicon substrate as a master for the layer (V) (Fig. 3 (V a)). Then a $250 \mu \mathrm{m}$ thick PDMS layer was coated on a SU-8 master. Since the coated PDMS layer was thinner than the SU-8 master, there were hollowed crescent patterns on this PDMS layer (Fig. 3 (V b)). A $1 \mu$ m thick SU-8 layer was deposited on the PDMS layer to enhance the adhesion for on-top microneedles. After that, a $16 \times 16 \mathrm{SU}-8$ pillars array was defined on the top of the PDMS layer through a standard UV lithography process (Fig. 3 (V c)). By drawing lithography technology we reported before [50], $500 \mu \mathrm{m}$ long sharp tips were integrated on the top of SU-8 pillars to form the $850 \mu \mathrm{m}$ long microneedles array (Fig. 3 (V c)). The spacing between two nearby microneedles is $500 \mu \mathrm{m}$. Drawing lithography technology is a maskless fabrication approach to build 3D structures based on the polymers' different viscosities under different temperatures. Briefly, a $200 \mu \mathrm{m}$ thick SU-8 layer was spun on a Si substrate and baked on the $95{ }^{\circ} \mathrm{C}$ hotplate to remove the solvent. Layer (V) with SU-8 pillars was fixed on a 3 dimensional precision stage and aligned above the baked SU-8 layer. By adjusting the precision stage, it was lowered down until the SU-8 pillars were immersed into the baked SU-8. The baked SU-8 encapsulated the pillars' surface due to its high viscosity. After that, the layer (V) with SU-8 pillars was drawn away from the baked SU-8. During the drawing process, both the temperature and drawing speed were increased. Since the SU-8 was less viscous at higher temperature, the connections between the SU-8 pillars and surface of the baked SU-8 became individual SU-8 bridges, shrank and then broke. The end of the shrunk SU-8 bridge finally formed a sharp tip on the top of each SU-8 pillar when the connection was separated. Subsequently, with a careful alignment, the layer (I) and layer $(\mathrm{V})$ were bonded with the layer containing microfluidic 


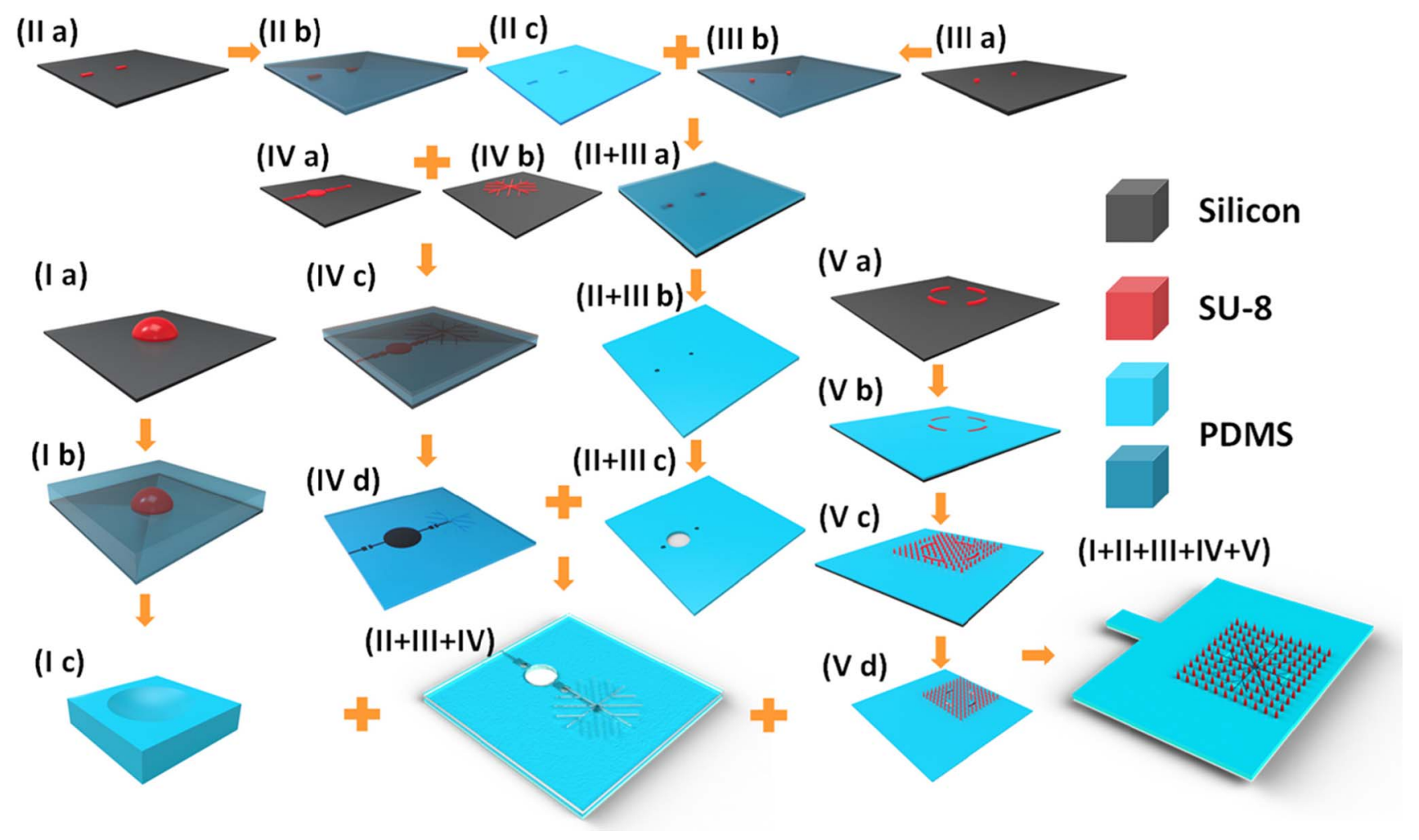

Fig. 3. Fabrication process for the proposed MFS. (I a) to (I c) are detailed process description for layer (I) fabrication. (II a) to (II c) are detailed process description for layer (II) fabrication. (III a) to (III b) are detailed process description for layer (III) fabrication. (IV a) to (IV d) are detailed process description for layer (IV) fabrication. ( $\mathrm{V}$ a) to $(\mathrm{V}$ d) are detailed process description for layer $(\mathrm{V})$ fabrication. $(\mathrm{I}+\mathrm{II}+\mathrm{III}+\mathrm{IV}+\mathrm{V})$ is the final device bonded from 5 layers.

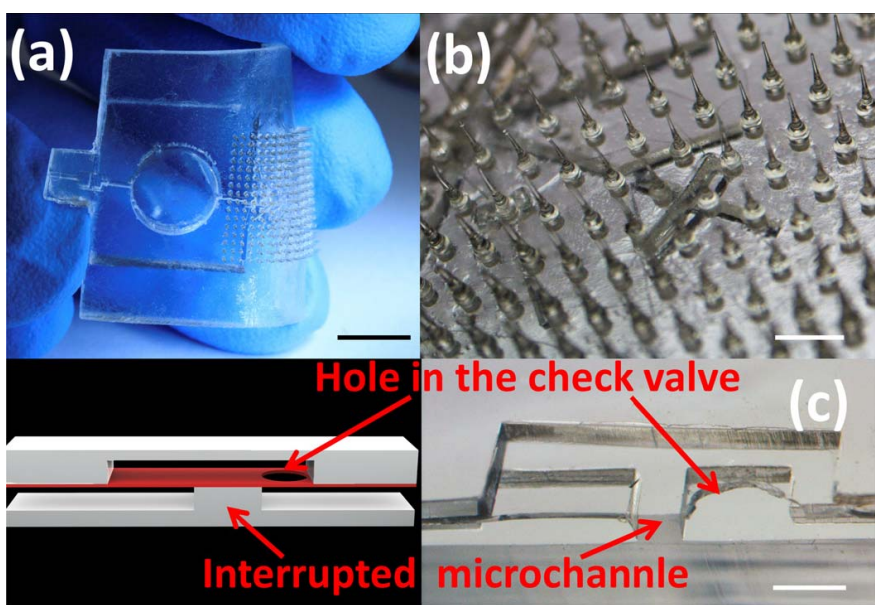

Fig. 4. (a) Optical image for the final flexible device. (Scale bar: $5 \mathrm{~mm}$ ) (b) Optical image for the crescent openings and microneedles on the top of MFS. (Scale bar: $500 \mu \mathrm{m}$ ) (c) Sectioned view of check valve in the MFS. (Scale bar: $1 \mathrm{~mm})$

check valves and dispensing network. The rear part of the chip was then cut to be a protruding beam structure for the easier drug loading purpose (Fig. 3 (I+II + III + IV + V)) The final device was shown in the Fig. 4. Three layers in the check valve are properly aligned (Fig. 4 (c)) and hollowed crescent patterns are successfully fabricated as openings for the underneath microfluidic dispensing networks (Fig. 4 (b)).

\section{CHARACTERIZATION}

\section{A. Characterization for Drug Loading and Delivery Volumes}

The finger-driven drug loading and delivery functions are demonstrated in Fig. 5 (a) and Fig. 5 (b). Methylene blue was added into the solution for a better inspection purpose. By pressing the micro pump with fingers, the outmost PDMS layers fully deformed and the air inside was pushed out, which led the pressure inside the chamber to be lower than the pressure in the air outside the chamber (Fig. 5 (a1)). The protruding beam structure was then lowered down to a container storing the desired drug (Fig. 5 (a2)). After releasing the outmost PDMS layers, the drug was driven to flow into the chamber through the inlet check valve from the loading port (Fig. 5 (a4)). By pressing the micro pump again, drug inside the chamber was driven to flow through outlet check valve while the inlet check valve was sealed by the fluid pressure. After flowing through the connection hole between top-side microfluidic network and bottom-side interrupted microfluidic channel, the loaded drug was dispensed to microfluidic network branches and delivered through the crescent openings on the top of the MFS (Fig. 5(b)).

In the operation process, both the maximum volume of loaded drug and the maximum volume of delivered drug were determined by the micro pump sizes. In order to quantify the relationship between the size of the micro pump and maximum volume, MFSs with different micro pump were fabricated. These devices were tested under the condition that their micro 


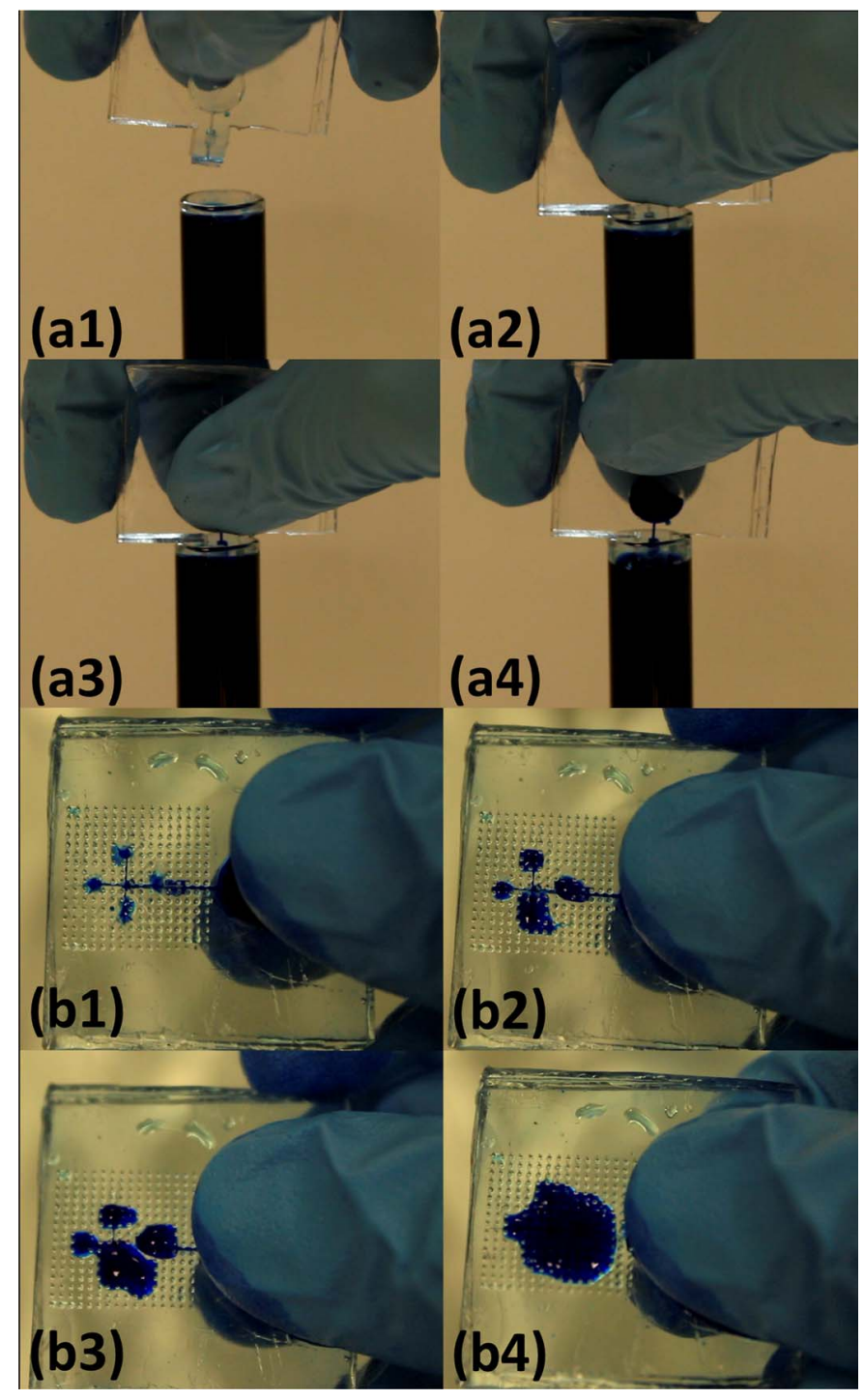

Fig. 5. (a) The finger-driven drug loading process in the fabricated MFS. (b) The finger-driven drug delivery process in the fabricated MFS.

pumps were fully deformed (the top cap of the chamber was deformed and in contact with the bottom substrate). The diameters of these micro pumps increased from $8 \mathrm{~mm}$ to $12 \mathrm{~mm}$ with $0.5 \mathrm{~mm}$ intervals (For each diameter, 5 pieces of devices were fabricated to calculate mean \pm S.E). When the drug was loaded inside or delivered outside the chamber, the weight of the fabricated MFS changed. By measuring the weight difference, the loaded volume and delivered volume were calculated and shown in Fig. 6.

When the micro pump became larger, both the loaded volume and delivered volume were found to keep increasing accordingly. Since the drug solution was normally an incompressible fluid, the volume of loaded and delivered drugs depended on the micro pump volume difference between different operation stages. Iwai et al. reported that when the micro pump was fully deformed, the remaining volume of the chamber did not vary significantly with the changes in the specific micro pump [44]. Thus the difference of the micro pump volume between the deformed state and initial state was

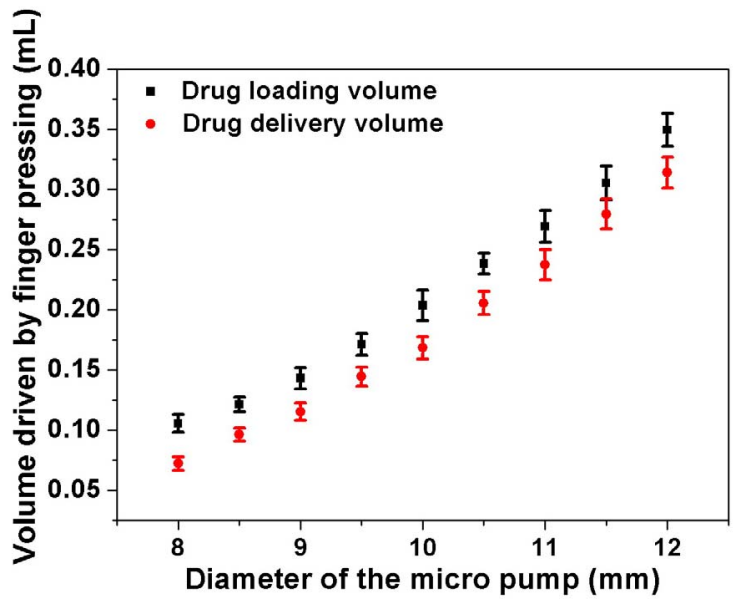

Fig. 6. The relationship between the sizes of the pressure chambers and maximum loading volume and maximum delivery volume.

mainly regulated by the volume of the micro pump with its original shape. It meant that the loaded and delivered volumes were mainly determined by the initial micro pump volume (shown as the diameter changing in Fig. 6). There was always some solution remained inside the microfluidic channel even the micro pump was fully deformed in the delivery process. That's why the delivered volume was slightly less than the loaded volume.

Except for proving the feasibility of the single push operation to load and deliver the drug, we also quantified the consistency of repeated push-and-release procedures by human fingers using multiple prototypes with micro pumps of $10 \mathrm{~mm}$ in diameter. Since the micro pump in microfluidic chip was more stable under longer operation duration [44], in each testing the micro pump was fully deformed by a long and slow push-and-release operation. The results were shown in Fig. 7. Each data point represents the maximum loaded volume and delivered volume during each push-and-release procedure. Among the 20 tests for 5 different prototypes, the maximum drug loading volume was $0.202 \mathrm{~mL} \pm 0.009 \mathrm{~mL}$ while the maximum delivery volume was $0.171 \mathrm{~mL} \pm 0.007 \mathrm{~mL}$. It shows that the fabricated MFS devices provide good consistency. The loaded and delivered volumes were found to remain relatively constant during the operations in different prototypes, which suggested that the fabricated MFS had sufficient reliability in volume control among different samples.

\section{B. Stiffness Testing and Penetration Testing for MFS}

Microneedles in the MFS were mainly used to create microchannels on the skin surface. To prove that the fabricated microneedles in the MFS were strong enough for successful penetration, their buckling force were tested. 10 different MFS chips were fabricated and loaded under an axial compression with Instron Microtester 5848 (Instron, USA). The equipment drove a single microneedle against a metal plate at a speed of $20 \mu \mathrm{m} / \mathrm{s}$ until the microneedle broke. During the process, the force and its corresponding displacement were recorded by a computer. Davis et al. had studied the failure point of microneedles and defined that 


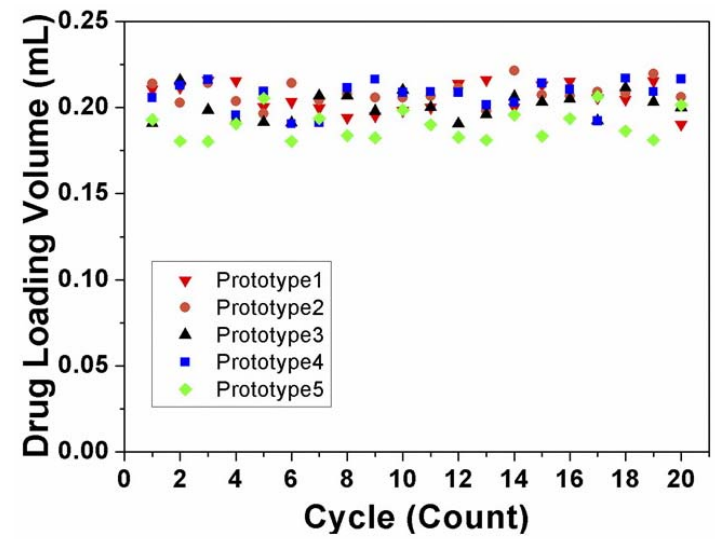

(a)

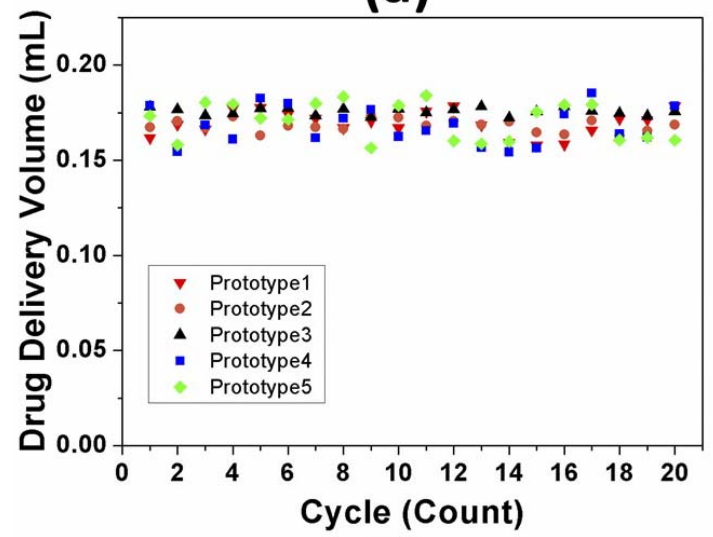

(b)

Fig. 7. Measurement for (a) loaded and (b) delivered volumes of the fabricated MFS (10 $\mathrm{mm}$ pressure chamber) in multiple prototypes during repeated push-and-release by human fingers.

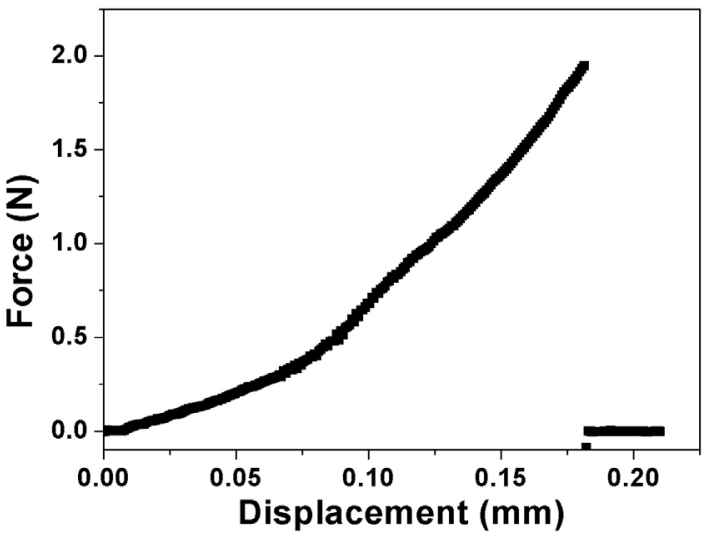

Fig. 8. A representative example of buckling force test result for a $500 \mu \mathrm{m}$ high microneedle.

the sharp discontinuity point marked the fracture of the microneedle [61]. We also followed this method to characterize the buckling force of fabricated MFS. Its failure loading point could be observed when the loading sensor output had a sharp change. Fig. 8 demonstrated one representative data of buckling force testing. When the metal plate touched the microneedle tip and lowered down, the axial force applied to a microneedle also increased. After the maximum load was reached, the microneedle

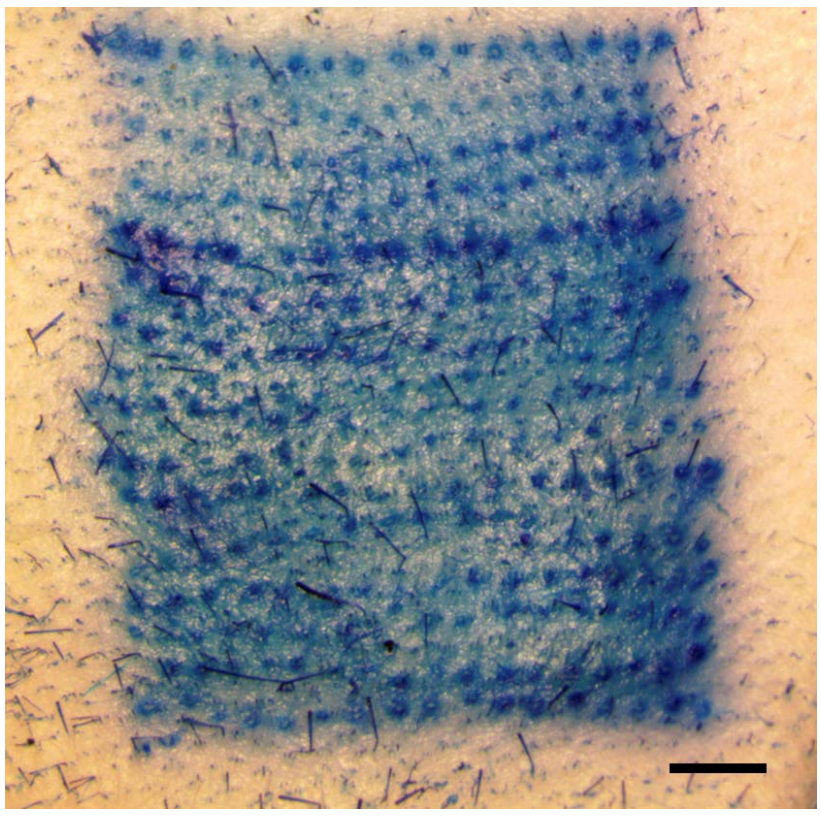

Fig. 9. Stained rat skin sample which was treated by fabricated MFS. The blue spots on the skin sample indicated the holes created by microneedles on the MFS (Scale bar : $1 \mathrm{~mm}$ )

broke and the metal plate automatically stopped moving. A discontinuity in the detected force in the graph indicated the fractured threshold force for the microneedle, which was also confirmed by visual observation during the test. By testing 100 microneedles from 10 MFS devices, the average buckling force was as much as $1.92 \mathrm{~N} \pm 0.16 \mathrm{~N}$, It was much larger than the minimal required penetration force (less than $0.5 \mathrm{~N}$ ) for the microneedles with similar geometry and dimension [38], which proved that the fabricated microneedles were strong enough to penetrate skin surface.

In order to further prove skin penetration capability of microneedles, after the MFS chip was applied to one rat's skin sample, $50 \mu \mathrm{L}$ of methylene blue solution was used to stain the skin surface. The stain was kept on the sample for 10 minutes for the methylene blue fully diffusing into tissues. The excess stain was then removed by ethanol wipes to show the stained pattern on the skin sample. Since the methylene blue solution only diffused into the penetrated holes, the stained dots revealed microchannels successfully created by the inserted microneedles. Fig. 9 showed one of the representative results. In the 10 tested MFS devices, all of them were successfully inserted into skin samples.

Histological images were also taken to show the details of microchannels created by these microneedles. Skin samples after penetration were washed in $1 \mathrm{X}$ phosphate buffered saline (PBS) (Vivantis Inc. USA) and fixed in 4\% paraformaldehyde (Electron Microscopy Sciences, USA) for 48 hours at $4{ }^{\circ} \mathrm{C}$. Then the samples were applied cryoprotection with 30\% Sucrose (Fischer Scientific, UK) for 24 hours at $4{ }^{\circ} \mathrm{C}$ and embedded with OCT medium. $20 \mu \mathrm{m}$ cryosections were sliced and dried at room temperature for 24 hours, stained with hematoxylin and eosin (H\&E) to locate the microneedle penetration in the skin. Fig. 10 shows 


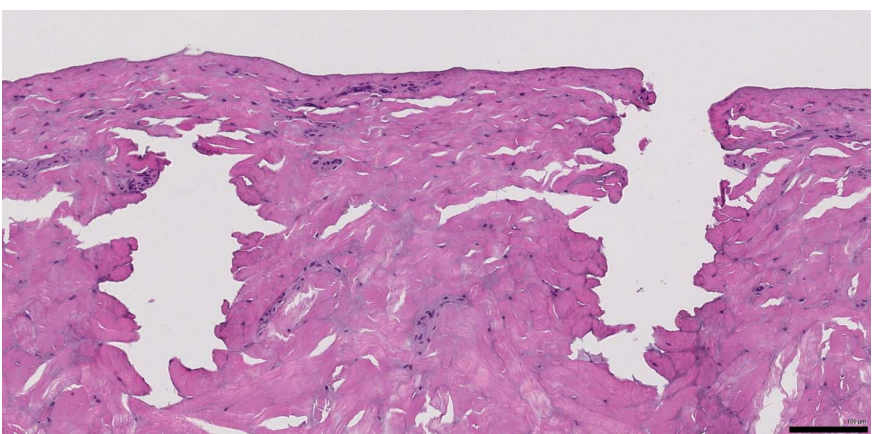

Fig. 10. Histology image of two microneedles penetration (Scale bar : $100 \mu \mathrm{m}$ ).

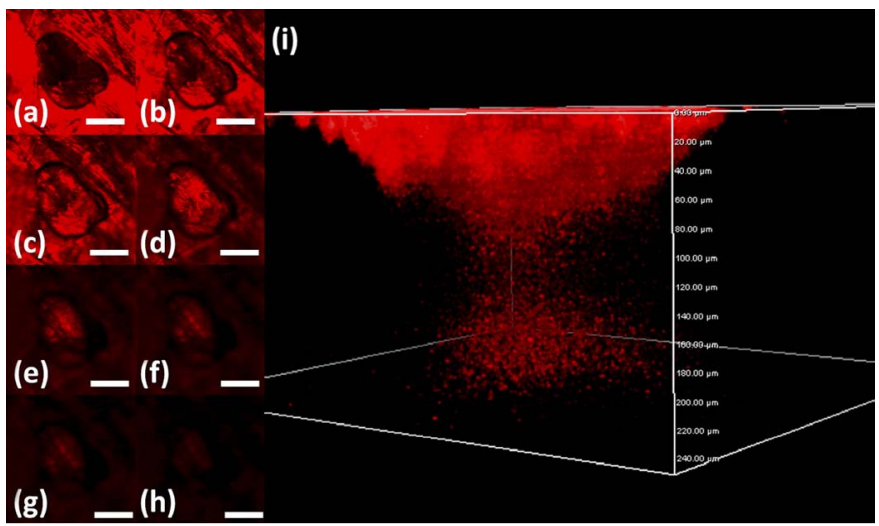

Fig. 11. Images of confocal microscopy of the site where one microneedle inserted shows that the fluorescent solution is delivered into the tissue underneath the skin surface. Optical section depths are (a) $0 \mu \mathrm{m}$, (b) $30 \mu \mathrm{m}$, (c) $60 \mu \mathrm{m}$, (d) $90 \mu \mathrm{m}$, (e) $120 \mu \mathrm{m}$, (f) $150 \mu \mathrm{m}$, (g) $180 \mu \mathrm{m}$, (h) $210 \mu \mathrm{m}$ below the skin surface. (Scale bar : $50 \mu \mathrm{m}$ ) (i) $3 \mathrm{D}$ confocal reconstruction image.

conical microchannels on the skin epidermis were created by penetration of microneedles for delivery purpose.

\section{Drug Diffusion Testing}

The presented data so far has only shown the functionality of integrated micro pump, check valves and microneedles, but not addressed the drug delivery capability. After the MFS was applied on rat skin, microchannles are created through SC layer because of microneedles. Then drug loaded inside the MFS could be pumped out by finger pressing and diffuse through these microchannles. In order to present this scenario, physiological saline solution containing Sulforhodamine (Sigma-Aldrich, Singapore) was loaded in the MFS and delivered into the rat skin sample. Then the skin sample was investigated by a confocal microscope. The permeation pattern of the florescent solution along the microchannels showed the solution diffusing results. Fig. 11 indicated the diffused area from the depth of $0 \mu \mathrm{m}$ to $210 \mu \mathrm{m}$ below the skin surface. The black area was a control area without any diffused florescence. In contrast, the illuminated tissues indicated the area where the solution diffused to. Since the microchannels were created by the conical microneedles, the diameter of microchannel decreased when the penetrated depth increased. Most Sulforhodamine solution was confined to the microchannles and only parts of them could diffuse to the nearby tissue. Thus, their diffusion area also decreased in the deeper region. Based on these layer by layer images, the $3 \mathrm{D}$ confocal reconstruction image showed the penetration depth and diffused florescent profile (Fig. 11 (g)).

\section{Transdermal Delivery of Diclofenac in Vivo}

Transdermal delivery of diclofenac was tested in vivo to prove the functionality of fabricated MFS to treat local inflammation disease. All the procedures were performed under protocol and approved by the Institutional Animal Care and Use Committee in the National University of Singapore.

Female Sprague-Dawley rats were divided into 3 groups (3 per group). Hair on the back of these rats was shaved by electric clippers. Then the rats were housed in polypropylene cage for 24 hours. The animals were maintained without access to food and water during the experiments. Meanwhile, the diclofenac solution was prepared by mixing $1 \%(\mathrm{w} / \mathrm{w})$ sodium diclofenac with $19 \%(\mathrm{w} / \mathrm{w})$ Transcutol and $80 \% 1 \times$ PBS. 3 minutes sonication was applied to the prepared solution for a full mixing purpose. Before the experiments, the rats were anaesthetized by katemine. To induce local inflammation, $50 \mu \mathrm{L} 1 \%$ carrageenan (w/v) in $1 \times$ PBS was injected into the plantar surface of the rats' hind paw at time zero. The group A was a control group without any other treatment. $150 \mu \mathrm{L}$ diclofenac solution was applied on the back of rats in group B, which was expected to diffuse into the tissue passively. A piece of parafilm covered the back of rats to avoid any evaporation. In group $\mathrm{C}$, MFS with $9.5 \mathrm{~mm}$ micro pump (calibrated with 146.56uL $\pm 5.96 \mu \mathrm{L}$ delivery volume in 10 samples) was fully loaded with diclofenac solution and applied on the back of rats. After fully pressed the micro pump on the MFS, diclofenac solution flowed through crescent openings, diffused into microchannels and was absorbed by tissues under the skin. The back of rats was also covered by a piece of parafilm and fixed with hypoallergenic sticking plaster to avoid any drug solution evaporation. The increment in paw metatarsal thickness was measured by a digital thickness gauge (Mitutoyo, Japan) at time 0, 1, 2, 3, 4, 5 and $6 \mathrm{~h}$ after carrageenan administration. The percentage of paw volume increment from time zero was calculated. The time-course of the anti-inflammatory effect was determined.

The results for anti-inflammatory activity were shown in Fig. 12. In the control group, the paw volume increments were $30.21 \%, 46.31 \%, 57.31 \%, 65.41 \%, 61.12 \%$ and $53.14 \%$ at $1,2,3,4,5$ and 6 hours after carrageenan administration. Since the rats in group 1 didn't receive any treatment, they showed the natural reaction of tissues to the induced carrageenan solution. In the group 2 (passive diffusion), the paw volume increments were $28.17 \%, 37.23 \%, 42.16 \%$, $45.31 \%, 40.32 \%$ and $36.14 \%$ while the paw volume increments were $19.12 \%, 28.16 \%, 35.16 \%, 27.63 \%, 25.61 \%$ and $24.39 \%$ in the group 3 (treated with MFS) at 1, 2, 3, 4, 5 and 6 hours after carrageenan administration. It was obvious that the rats had anti-inflammatory activity in both group 2 and group 3 . However, the paw volume increment was less in group 3 (treated with MFS) than that in group 2 (passive diffusion). It demonstrated that the anti-inflammatory 


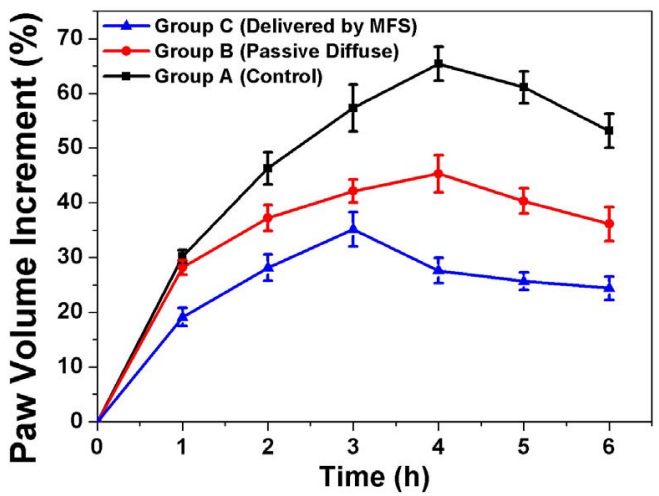

Fig. 12. Percentage increment of paw volume after subplantar injection of carrageenan in control group A (ם), in group B with diclofenac passive diffusion ( $\bullet$ ) and in group $\mathrm{C}$ with diclofenac delivered by MFS ( $\boldsymbol{\Delta})$, respectively. Results are the mean \pm S.E. for 3 rats.

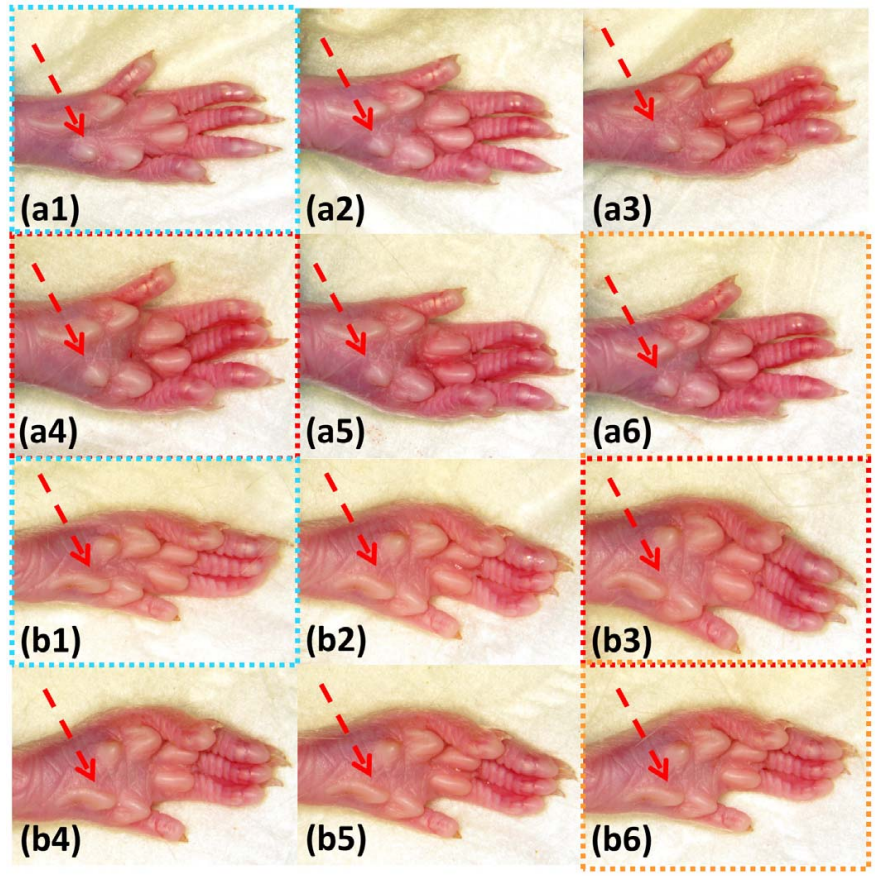

Fig. 13. Six representative example images for the paw swelling in a rat of (a) group B with diclofenac passive diffusion and (b) group $\mathrm{C}$ with diclofenac delivered by MFS. (a1),(b1) Initial state of the rat's paw (in blue dashed box); 2 hours after diclofenac solution was (a2) applied on the back of rats and (b2) delivered by MFS; (a3) 3 hours after diclofenac solution was applied on the back of rats; (b3) The maximum paw volume at hour 3 after diclofenac solution was delivered by MFS (in red dashed box); (a4) The maximum paw volume at hour 4 after diclofenac solution was applied on the back of rats (in red dashed box); (b4) 4 hours after diclofenac solution was delivered by MFS; 5 hours after diclofenac solution was (a5) applied on the back of rats and (b5) delivered by MFS; The recovered status at hour 6 after diclofenac solution was (a6) applied on the back of rats and (b6) delivered by MFS (in orange dashed box). The red arrows indicate the location of paw volume increment measurement.

activity was more significant when the rats were treated by the fabricated MFS. Moreover, in both group 1 (control group) and group 2 (passive diffusion), the maximum paw volume appeared at hour 4 (one representative example was shown in Fig. 13 (a4)). However, in group 3 (treated with MFS), the maximum paw volume time point came at hour 3 (one representative example was shown in Fig. 13 (b3)). It showed that after treated with MFS, the inflammation activity was inhibited earlier than the inflammation in rats who received diclofenac solution just by passive diffusion. The in vivo testing successfully proved the capability of fabricated MFS to deliver diclofenac solution for more significant and efficient treatment to local inflammation disease.

\section{CONCLusion}

A disposable and flexible microneedle-fluidic-system (MFS) device is developed to achieve finger driven on-chip drug loading and delivery functions by integrating Polydimethylsiloxane (PDMS) based microfluidic dispensing networks, check valves, micro pump and microneedles on a single device. Drugs loaded in the MFS by micro pump are driven to pass the outlet check valve, flow to the top-side microfluidic network through the connection hole, dispense to microfluidic network branches and finally deliver through the crescent openings. These drugs then permeate through the microchannels created by microneedles and are delivered into tissues under the skin. The loading and delivery volumes are determined by the micro pump size. Consistency and reliability testing proved the fabricated MFS device is able to control the loading and deliver volume. The in vitro mechanical testing, penetration testing and delivery testing prove the device functionalities. The in vivo testing successfully proved the capability of fabricated MFS device to deliver diclofenac solution for more significant and efficient treatment to local inflammation disease.

\section{REFERENCES}

[1] R. Singh, S. Singh, and J. W. Lillard, "Past, present, and future technologies for oral delivery of therapeutic proteins," J. Pharm. Sci., vol. 97, no. 7, pp. 2497-2523, 2008.

[2] Y. Nir, A. Paz, E. Sabo, and I. Potasman, "Fear of injections in young adults: Prevalence and associations," Amer. J. Tropical Med. Hygiene, vol. 68, no. 3, pp. 341-344, 2003.

[3] G. Cevc, G. Blume, A. Schätzlein, D. Gebauer, and A. Paul, "The skin: A pathway for systemic treatment with patches and lipid-based agent carriers," Adv. Drug Del. Rev., vol. 18, no. 3, pp. 349-378, 1996.

[4] M. R. Prausnitz, S. Mitragotri, and R. Langer, "Current status and future potential of transdermal drug delivery," Nature Rev. Drug Discovery, vol. 3, no. 2, pp. 115-124, 2004.

[5] J. D. Bos and M. M. H. M. Meinardi, "The 500 Dalton rule for the skin penetration of chemical compounds and drugs," Experim. Dermatol., vol. 9 , no. 3, pp. 165-169, 2000.

[6] B. C. Finnin and T. M. Morgan, "Transdermal penetration enhancers: Applications, limitations, and potential," J. Pharm. Sci., vol. 88, no. 10, pp. 955-958, 1999.

[7] A. Nayak, D. B. Das, and G. T. Vladisavljević, "Microneedle-assisted permeation of lidocaine carboxymethylcellulose with gelatine copolymer hydrogel," Pharm. Res., vol. 31, no. 5, pp. 1170-1184, 2013.

[8] A. Nayak, H. Babla, T. Han, and D. B. Das, "Lidocaine carboxymethylcellulose with gelatine co-polymer hydrogel delivery by combined microneedle and ultrasound," Drug Deliv., In press.

[9] M. R. Prausnitz, "Microneedles for transdermal drug delivery," $A d v$. Drug Del. Rev., vol. 56, no. 5, pp. 581-587, 2004.

[10] Y.-G. Lv, J. Liu, Y.-H. Gao, and B. Xu, "Modeling of transdermal drug delivery with a microneedle array," J. Micromech. Microeng., vol. 16, no. 11, pp. 2492-2501, 2006.

[11] A. Arora, M. R. Prausnitz, and S. Mitragotri, "Micro-scale devices for transdermal drug delivery," Int. J. Pharm., vol. 364, no. 2, pp. 227-236, 2009.

[12] R. F. Donnelly, T. R. R. Singh, and A. D. Woolfson, "Microneedle-based drug delivery systems: Microfabrication, drug delivery, and safety," Drug Del., vol. 17, no. 4, pp. 187-207, 2010. 
[13] S. Kaushik et al., "Lack of pain associated with microfabricated microneedles," Anesthesia Analgesia, vol. 92, no. 2, pp. 502-504, 2001.

[14] R. K. Sivamani, B. Stoeber, G. C. Wu, H. Zhai, D. Liepmann, and H. Maibach, "Clinical microneedle injection of methyl nicotinate: Stratum corneum penetration," Skin Res. Technol., vol. 11, no. 2, pp. 152-156, 2005.

[15] T. Omatsu et al., "Metal microneedle fabrication using twisted light with spin," Opt. Exp., vol. 18, no. 17, pp. 17967-17973, 2010

[16] B. P. Chaudhri, F. Ceyssens, P. De Moor, C. Van Hoof, and R. Puers, "A high aspect ratio SU-8 fabrication technique for hollow microneedles for transdermal drug delivery and blood extraction," J. Micromech. Microeng., vol. 20, no. 6, p. 064006, 2010.

[17] Z. Xiang, H. Wang, A. Pant, G. Pastorin, and C. Lee, "Development of vertical SU-8 microtubes integrated with dissolvable tips for transdermal drug delivery," Biomicrofluidics, vol. 7, no. 2, p. 026502, 2013.

[18] Z. Xiang, H. Wang, A. Pant, G. Pastorin, and C. Lee, "Development of vertical SU-8 microneedles for transdermal drug delivery by double drawing lithography technology," Biomicrofluidics, vol. 7, no. 6, p. 066501, 2013.

[19] Z. Xiang, H. Wang, S. K. Murugappan, S.-C. Yen, G. Pastorin, and C. Lee, "Dense vertical SU-8 microneedles drawn from a heated mold with precisely controlled volume," J. Micromech. Microeng., vol. 25 , no. 2, p. 025013, 2015.

[20] Y. Liu, N. J. Bowen, L. Matyunina, J. McDonald, and M. R. Prausnitz, "Gene transfection enhanced by ultrasound exposure combined with drug treatment guided by gene chip analysis," Int. J. Hyperthermia, vol. 28, no. 4, pp. 349-361, 2012.

[21] J. J. Norman, J. M. Arya, M. A. McClain, P. M. Frew, M. I. Meltzer, and M. R. Prausnitz, "Microneedle patches: Usability and acceptability for self-vaccination against influenza," Vaccine, vol. 32, no. 16, pp. 1856-1862, 2014

[22] H. J. G. E. Gardeniers et al., "Silicon micromachined hollow microneedles for transdermal liquid transport," J. Microelectromech. Syst., vol. 12, no. 6, pp. 855-862, 2003.

[23] W. Martanto, S. P. Davis, N. R. Holiday, J. Wang, H. S. Gill, and M. R. Prausnitz, "Transdermal delivery of insulin using microneedles in vivo," Pharm. Res., vol. 21, no. 6, pp. 947-952, 2004.

[24] O. Olatunji, D. B. Das, M. J. Garland, L. Belaid, and R. F. Donnelly, "Influence of array interspacing on the force required for successful microneedle skin penetration: Theoretical and practical approaches," J. Pharm. Sci., vol. 102, no. 4, pp. 1209-1221, 2013.

[25] K. Cheung, T. Han, and D. B. Das, "Effect of force of microneedle insertion on the permeability of insulin in skin," J. Diabetes Sci. Technol., vol. 8, no. 3, pp. 444-452, 2014.

[26] N. Wilke, A. Mulcahy, S.-R. Ye, and A. Morrissey, "Process optimization and characterization of silicon microneedles fabricated by wet etch technology," Microelectron. J., vol. 36, no. 7, pp. 650-656, 2005.

[27] N. Wilke and A. Morrissey, "Silicon microneedle formation using modified mask designs based on convex corner undercut," J. Micromech. Microeng., vol. 17, no. 2, pp. 238-244, 2007.

[28] H. S. Gill and M. R. Prausnitz, "Coated microneedles for transdermal delivery," J. Controlled Release, vol. 117, no. 2, pp. 227-237, 2007.

[29] Y.-C. Kim, F.-S. Quan, R. W. Compans, S.-M. Kang, and M. R. Prausnitz, "Formulation and coating of microneedles with inactivated influenza virus to improve vaccine stability and immunogenicity," J. Controlled Release, vol. 142, no. 2, pp. 187-195, 2010

[30] C. Y. Jin, M. H. Han, S. S. Lee, and Y. H. Choi, "Mass producible and biocompatible microneedle patch and functional verification of its usefulness for transdermal drug delivery," Biomed. Microdevices, vol. 11, no. 6, pp. 1195-1203, 2009.

[31] I. Mansoor, Y. Liu, U. O. Häfeli, and B. Stoeber, "Arrays of hollow outof-plane microneedles made by metal electrodeposition onto solvent cast conductive polymer structures," J. Micromech. Microeng., vol. 23, no. 8, p. 085011, 2013.

[32] R. F. Donnelly et al., "Design, optimization and characterisation of polymeric microneedle arrays prepared by a novel laser-based micromoulding technique," Pharm. Res., vol. 28, no. 1, pp. 41-57, 2011.

[33] A. Nayak and D. B. Das, "Potential of biodegradable microneedles as a transdermal delivery vehicle for lidocaine," Biotechnol. Lett., vol. 35 , no. 9, pp. 1351-1363, 2013.

[34] K. Cheung and D. B. Das, "Microneedles for drug delivery: Trends and progress," Drug Deliv., In press.

[35] S. Y. Yang et al., "A bio-inspired swellable microneedle adhesive for mechanical interlocking with tissue," Nature Commun., vol. 4, Apr. 2013, Art. ID 1702
[36] K. A. Moga et al., "Rapidly-dissolvable microneedle patches via a highly scalable and reproducible soft lithography approach," Adv. Mater. vol. 25 , no. 36, pp. 5060-5066, 2013.

[37] P.-C. Wang, S.-J. Paik, S. Chen, S. Rajaraman, S.-H. Kim, and M. G. Allen, "Fabrication and characterization of polymer hollow microneedle array using uv lithography into micromolds," J. Microelectromech. Syst., vol. 22, no. 5, pp. 1041-1053, Oct. 2013.

[38] P.-C. Wang, S.-J. Paik, S.-H. Kim, and M. G. Allen, "Hypodermicneedle-like hollow polymer microneedle array: Fabrication and characterization," J. Microelectromech. Syst., vol. 23, no. 4, pp. 991-998, 2014.

[39] K. Kim and J.-B. Lee, "High aspect ratio tapered hollow metallic microneedle arrays with microfluidic interconnector," Microsyst. Technol., vol. 13, nos. 3-4, pp. 231-235, 2006.

[40] B. Ma et al., "A PZT insulin pump integrated with a silicon microneedle array for transdermal drug delivery," Microfluidics Nanofluidics, vol. 2, no. 5, pp. 417-423, 2006

[41] N. Roxhed, B. Samel, L. Nordquist, P. Griss, and G. Stemme, "Painless drug delivery through microneedle-based transdermal patches featuring active infusion," IEEE Trans. Biomed. Eng., vol. 55, no. 3, pp. 1063-1071, Mar. 2008.

[42] C. Mousoulis, M. Ochoa, D. Papageorgiou, and B. Ziaie, "A skincontact-actuated micropump for transdermal drug delivery," IEEE Trans. Biomed. Eng., vol. 58, no. 5, pp. 1492-1498, May 2011.

[43] W. Li et al., "Squeeze-chip: A finger-controlled microfluidic flow network device and its application to biochemical assays," Lab Chip, vol. 12, no. 9, pp. 1587-1590, 2012.

[44] K. Iwai, K. C. Shih, X. Lin, T. A. Brubaker, R. D. Sochol, and L. Lin, "Finger-powered microfluidic systems using multilayer soft lithography and injection molding processes," Lab Chip, vol. 14, no. 19, pp. 3790-3799, 2014

[45] C.-H. Wang and G.-B. Lee, "Automatic bio-sampling chips integrated with micro-pumps and micro-valves for disease detection," Biosensors Bioelectron., vol. 21, no. 3, pp. 419-425, 2005.

[46] S.-H. Chiu and C.-H. Liu, "An air-bubble-actuated micropump for onchip blood transportation," Lab Chip, vol. 9, no. 11, pp. 1524-1533, 2009.

[47] E. L. Fosbøl et al., "Cause-specific cardiovascular risk associated with nonsteroidal antiinflammatory drugs among healthy individuals," Circulat. Cardio. Qual. Outcomes, vol. 3, no. 4, pp. 395-405, 2010.

[48] P. McGettigan and D. Henry, "Cardiovascular risk with non-steroidal anti-inflammatory drugs: Systematic review of population-based controlled observational studies," PLoS Med., vol. 8, no. 9, p. e1001098, 2011.

[49] L. G. Rodríguez and H. Jick, "Risk of upper gastrointestinal bleeding and perforation associated with individual non-steroidal anti-inflammatory drugs," Lancet, vol. 343, no. 8900, pp. 769-772, 1994.

[50] J.-R. Laporte, X. Carné, X. Vidal, V. Moreno, and J. Juan, "Upper gastrointestinal bleeding in relation to previous use of analgesics and non-steroidal anti-inflammatory drugs. Catalan countries study on upper gastrointestinal bleeding," Lancet, vol. 337, no. 8733, pp. 85-89, 1991.

[51] N. K. Brogden, S. L. Banks, L. J. Crofford, and A. L. Stinchcomb, "Diclofenac enables unprecedented week-long microneedle-enhanced delivery of a skin impermeable medication in humans," Pharm. Res., vol. 30, no. 8, pp. 1947-1955, 2013.

[52] P. Ghosh, D. Lee, K. B. Kim, and A. L. Stinchcomb, "Optimization of naltrexone diclofenac codrugs for sustained drug delivery across microneedle-treated skin," Pharm. Res., vol. 31, no. 1, pp. 148-159, 2014.

[53] N. K. Brogden, M. Milewski, P. Ghosh, L. Hardi, L. J. Crofford, and A. L. Stinchcomb, "Diclofenac delays micropore closure following microneedle treatment in human subjects," J. Controlled Release, vol. 163, no. 2, pp. 220-229, 2012.

[54] M. A. Unger, "Monolithic microfabricated valves and pumps by multilayer soft lithography," Science, vol. 288, no. 5463, pp. 113-116, 2000.

[55] E. Seker, D. C. Leslie, H. Haj-Hariri, J. P. Landers, M. Utz, and M. R. Begley, "Nonlinear pressure-flow relationships for passive microfluidic valves," Lab Chip, vol. 9, no. 18, pp. 2691-2697, 2009.

[56] D. C. Leslie et al., "Frequency-specific flow control in microfluidic circuits with passive elastomeric features," Nature Phys., vol. 5, no. 3, pp. 231-235, 2009

[57] B. Mosadegh, H. Tavana, S. C. Lesher-Perez, and S. Takayama, "High-density fabrication of normally closed microfluidic valves by patterned deactivation of oxidized polydimethylsiloxane," Lab Chip, vol. 11 , no. 4 , pp. $738-742,2011$. 
[58] B. Mosadegh et al., "Integrated elastomeric components for autonomous regulation of sequential and oscillatory flow switching in microfluidic devices," Nature Phys., vol. 6, no. 6, pp. 433-437, 2010.

[59] B.-H. Jo, L. M. Van Lerberghe, K. M. Motsegood, and D. J. Beebe, "Three-dimensional micro-channel fabrication in polydimethylsiloxane (PDMS) elastomer," J. Microelectromech. Syst., vol. 9, no. 1, pp. 76-81, 2000.

[60] N. Lucas, S. Demming, A. Jordan, P. Sichler, and S. Büttgenbach, "An improved method for double-sided moulding of PDMS," J. Micromech. Microeng., vol. 18, no. 7, p. 075037, 2008.

[61] S. P. Davis, B. J. Landis, Z. H. Adams, M. G. Allen, and M. R. Prausnitz, "Insertion of microneedles into skin: Measurement and prediction of insertion force and needle fracture force," J. Biomech., vol. 37, no. 8, pp. 1155-1163, 2004.

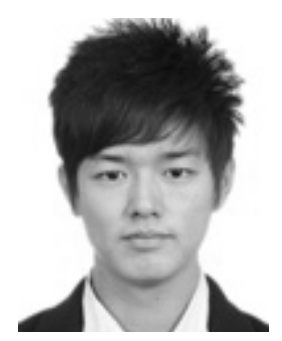

Zhuolin Xiang received the B.Eng. degree from the Department of Information and Electronics, Beijing Institute of Technology, Beijing, China, in 2011. He is currently pursuing the Ph.D. degree in electrical and computer engineering with the National University of Singapore. His research interests focus mainly on BioMEMS devices for drug delivery and neural interfacing.

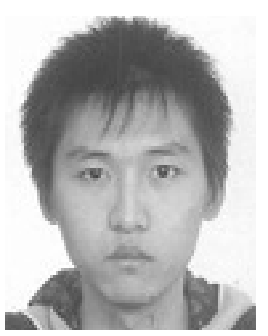

Hao Wang received the B.Eng. degree from the School of Optoelectronic Information, University of Electronic Science and Technology of China, in 2010. He is currently pursuing the M.Eng. degree with the Department of Electrical and Computer Engineering. $\mathrm{He}$ is also a Research Engineer of Electrical and Computer Engineering with the National University of Singapore. His research interests are focused on nanoneedle devices for transdermal drug delivery.

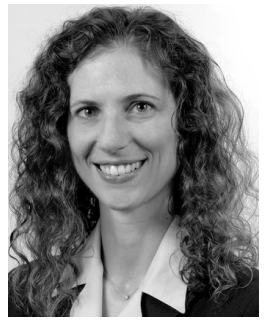

Giorgia Pastorin received the Ph.D. degree in medicinal chemistry in Italy in 2004. She specialized in drug delivery through the use of functionalized nanomaterials for several biomedical applications. She is currently an Associate Professor with the Department of Pharmacy, National University of Singapore, where she is responsible for the Bio-Laboratory in nanocore. She has authored over 70 research papers and reviews in internationally recognized journals, including Nature Nanotechnology, Proceedings of the National Academy of Sciences, Nano Letters, and Angewandte Chemie International Edition.

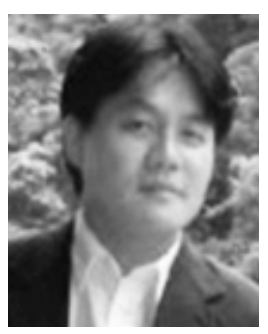

Chengkuo Lee (S'93-M'96) received the M.S. degree in materials science and engineering from National Tsing Hua University, Hsinchu, Taiwan, in 1991; the M.S. degree in industrial and system engineering from Rutgers University, New Brunswick, NJ, in 1993; and the Ph.D. degree in precision engineering from the University of Tokyo, Tokyo, Japan, in 1996.

He was a Foreign Researcher with the Nanometer Scale Manufacturing Science Laboratory, Research Center for Advanced Science and Technology, University of Tokyo, from 1993 to 1996. He was with the Mechanical Engineering Laboratory, AIST, MITI, Japan, as a JST Research Fellow, in 1996. Thereafter, he became a Senior Research Staff Member with the Microsystems Laboratory, Industrial Technology Research Institute, Hsinchu. In 1997, he joined Metrodyne Microsystem Corporation, Hsinchu, and established the MEMS Device Division and the first micromachining fab for commercial purposes in Taiwan. He was the Manager of the MEMS Device Division from 1997 to 2000. He was an Adjunct Assistant Professor with the Electro-Physics Department, National Chiao Tung University, Hsinchu, in 1998, and the Institute of Precision Engineering, National Chung Hsing University, Taichung, Taiwan, from 2001 to 2005. In 2001, he co-founded Asia Pacific Microsystems, Inc., where he first became the Vice President of Research and Development, before becoming the Vice President of the Optical Communication Business Unit and a Special Assistant to the Chief Executive Officer in charge of international business and technical marketing for the microelectromechanical systems (MEMS) foundry service. From 2006 to 2009, he was a Senior Member of the Technical Staff with the Institute of Microelectronics, A-STAR, Singapore. He is currently an Associate Professor with the Department of Electrical and Computer Engineering, National University of Singapore, Singapore. He has co-authored the books Advanced MEMS Packaging (McGraw-Hill, 2010) and Micro and Nano Energy Harvesting Technologies (Artech House, 2014). He has contributed to more than 240 international conference papers and extended abstracts, and 170 peer-reviewed international journal articles in the fields of sensors, actuators, energy harvesting, MEMS, nanoelectromechanical systems, metamaterials, nanophotonics, and nanotechnology. He holds nine U.S. patents. 